\title{
Lidil
}

Revue de linguistique et de didactique des langues

Acquisition et enseignement de la morphographie

\section{Le traitement morphologique en lecture chez des sourds gestuels québécois}

Daniel Daigle et Françoise Armand

\section{(2) OpenEdition}

\section{Journals}

Édition électronique

URL : http://journals.openedition.org/lidil/843

DOI : $10.4000 /$ lidil. 843

ISSN : $1960-6052$

\section{Éditeur}

UGA Éditions/Université Grenoble Alpes

\section{Édition imprimée}

Date de publication : 1 décembre 2004

Pagination : 117-131

ISBN : 2-914176-11-2

ISSN : 1146-6480

\section{Référence électronique}

Daniel Daigle et Françoise Armand, « Le traitement morphologique en lecture chez des sourds gestuels québécois », Lidil [En ligne], 30 | 2004, mis en ligne le 29 janvier 2008, consulté le 04 mai 2019. URL : http://journals.openedition.org/lidil/843; DOI : 10.4000/lidil.843 


\title{
LE TRAITEMENT MORPHOLOGIQUE EN LECTURE CHEZ DES SOURDS GESTUELS QUÉBÉCOIS
}

\author{
Daniel DAIGLE* \\ Françoise ARMAND**
}

Les problèmes d'apprentissage de la lecture chez les sourds constituent un obstacle majeur à leur réussite scolaire (Paul, 1998). Une des sources de difficultés des sourds en lecture se rapporte au traitement des mots (Paul, 1998 ; Transler, 1999). Or, sans un traitement rapide et automatisé des mots lors de leur reconnaissance, la réussite de l'apprentissage de la lecture peut être compromise (Stanovich, 1990).

Nous ne reprenons pas l'argumentation en faveur du rôle essentiel de la reconnaissance des mots en lecture (voir à ce sujet Baccino et Colé, 1995 ; et Sprenger-Charolles et Casalis, 1996). Notons cependant que lors de la reconnaissance des mots, différents processus phonologiques et orthographiques sont mis en œuvre. Ces processus, dont le traitement morphologique fait partie, facilitent l'activation de représentations lexicales qui permettent l'accès au sens des mots (pour une description des mécanismes de traitement des mots, voir, entre autres, Gombert, 1997).

* Daniel Daigle, Université de Montréal, Département de didactique, CP 6128, succursale centre-ville, Montréal (Québec) Canada, H3C 3J7, courriel : <daniel.daigle@umontreal.ca>

* Françoise Armand, Université de Montréal.

Nous tenons à remercier grandement Elisabeth Demont pour ses nombreux commentaires sur les premières versions de cet article. 


\section{Le traitement morphologique}

Chez les lecteurs adultes entendants, un certain nombre d'études plaident en faveur d'un traitement morphologique. On retient de ces études que la fréquence de surface (la fréquence d'un mot dans la langue écrite) et la fréquence de base (la fréquence totale des mots partageant la même racine dans la langue écrite) ont une incidence sur la lecture de mots en termes de temps de réponse et de réussite (Burani et al., 1984 ; Burani et Caramazza, 1987 ; Colé et al., 1989 ; Pollatsek et al., 2000). De plus, un effet de légalité morphologique (voir exemple ci-après) a été relevé dans plusieurs études (entre autres Beauvillain, 1994; Taft, 1994). Ainsi, lors d'une tâche de décision lexicale, c'est-à-dire lorsqu'on demande aux sujets de déterminer si une suite de lettres constitue ou non un mot, cet effet indique que des mots affixés (revoir) sont reconnus plus rapidement et mieux que des mots pseudoaffixés (retour), et que les pseudo-mots constitués en partie à partir d'un morphème existant (danaitre qui inclut naitre) sont rejetés, en tant que mots, plus lentement et occasionnent plus d'erreurs que les pseudo-mots n'incluant pas de morphème existant dans la langue (danoitre).

Chez de jeunes normo-lecteurs entendants, quelques études ont tenté, d'une part, d'évaluer la présence d'un traitement morphologique à des âges différents et, d'autre part, d'établir le lien qu'entretiennent le traitement morphologique et la compétence en lecture. A l'aide d'une tâche de probabilité lexicale où il est demandé aux sujets de déterminer si une suite de lettres est probable dans la langue écrite, Burani $e t$ al. (2002) concluent que les lecteurs italiens de 8 à 10 ans de leur étude croient plus probables les pseudo-mots morphologiquement légaux que les pseudo-mots qui ne respectent les règles de formation des mots. Ces résultats ont été interprétés en faveur de la présence d'une sensibilité à la légalité morphologique. À partir d'une tâche de lecture à voix haute, l'étude de Laxon et al. (1992) a montré chez de jeunes lecteurs anglophones de 7 à 9 ans que les mots affixés occasionnent moins d'erreurs que les mots pseudo-affixés et que le taux de réussite pour les pseudo-mots formés à partir d'une racine exis- 
tante dans la langue (gooder) était plus élevé que celui pour les pseudo-mots sans racine réelle (horter). À l'aide de différentes épreuves évaluant la sensibilité à la morphologie flexionnelle (4 épreuves) et à la morphologie dérivationnelle (5 épreuves), Casalis et Louis-Alexandre (2000) ont mené une étude réalisée auprès d'apprentis-lecteurs francophones de la maternelle à la $2^{\mathrm{e}}$ année du primaire. Les résultats de cette étude révèlent que la capacité à prendre en compte les indices morphologiques rend compte, en partie, de la réussite en lecture en $2^{e}$ année. Singson et al. (2000) ont mené une étude auprès de lecteurs anglophones de la $3^{\mathrm{e}}$ année à la $6^{\mathrm{e}}$ année du primaire à l'aide d'une tâche où il était demandé de déterminer parmi quatre mots ou quatre pseudo-mots variant en fonction de la structure morphologique lequel était le plus probable pour une phrase donnée. Les résultats indiquent que la réussite croît avec l'âge et les analyses de régression démontrent que le taux de succès à cette épreuve rend compte en partie du niveau de lecture à partir de la $4^{c}$ année. Enfin, Yu-Min et Anderson (2003) ont réalisé une étude auprès de jeunes lecteurs du chinois et de jeunes lecteurs de l'anglais de la $2^{\mathrm{e}}$, de la $4^{\mathrm{e}}$ et de la $6^{\mathrm{e}}$ année du primaire. Les auteurs ont eu recours à différentes épreuves : reconnaissance morphologique, discrimination de morphèmes, interprétation d'items polymorphémiques et jugement de probabilité lexicale. Les résultats des analyses de variance montrent, pour chaque épreuve, que plus les sujets sont âgés, plus la réussite est grande. Pour tous les sujets, la capacité à prendre en compte les indices morphologiques est liée à la réussite en lecture.

Les études abordées jusqu'ici ont été réalisées auprès de jeunes normo-lecteurs qui par ailleurs avaient des langues maternelles différentes. Abu-Rabia et al. (2003) se sont intéressés à des lecteurs en difficulté et ont eu recours à une batterie de tests mesurant notamment les traitements syntaxique, morphologique et phonologique. Les performances des sujets ayant un retard en lecture ont été comparées à celles de normo-lecteurs du même âge et à celles de lecteurs plus jeunes ayant le même niveau de lecture, tous arabophones. Les résultats indiquent que le meilleur prédicteur du niveau de lecture est le traitement phonologique suivi du traitement morphologique. De plus, ce qui différencie les lecteurs en dif- 
ficulté des deux autres groupes de sujets se rapporte à une moins grande capacité à manipuler la structure phonologique des items écrits et non à la capacité à considérer les indices morphologiques. Ceci amène les auteurs à penser que les lecteurs en difficulté pallient leur retard en lecture en ayant recours à des stratégies visuo-orthographiques.

Une seule étude a abordé, à notre connaissance, le traitement morphologique chez les sourds. Il s'agit de l'étude de Hanson et Wilkenfeld (1985) menée auprès de sujets sourds universitaires à l'aide d'une tâche de décision lexicale avec amorce (un item-amorce est présenté rapidement suivi de l'item-cible sur lequel doit porter la décision lexicale). Les items (think) étaient précédés soit d'une amorce morphologiquement liée (thought), soit d'une amorce orthographiquement semblable (thin). Les résultats révèlent que les mots précédés d'une amorce liée morphologiquement étaient reconnus plus rapidement que les mots précédés d'une amorce semblable orthographiquement. Ces résultats ont été interprétés en faveur d'un effet du traitement morphologique. De plus, cet effet serait plus marqué chez les lecteurs sourds dont le niveau de lecture est plus élevé.

Aucune étude n'a étudié le rôle du traitement morphologique en lecture chez de jeunes lecteurs sourds. Une meilleure connaissance de cette composante du traitement de l'écrit chez les sourds est susceptible de guider la mise en place de nouvelles pratiques pédagogiques pouvant faciliter l'enseignement de la lecture aux sourds. La présente étude a donc pour objectif de différencier entre eux des sujets sourds gestuels québécois sur la base de l'âge et du niveau de lecture, à partir d'une épreuve de jugement de probabilité lexicale. Évaluer la probabilité d'un pseudo-mot construit suivant les règles de formation des mots peut rendre possible l'observation de la présence d'un traitement morphologique par le lecteur. En effet, ce type d'épreuve permet d'évaluer la conscience qu'ont les sujets des règles de formation des mots. Par exemple, en français, le suffixe -able se juxtapose généralement à une base verbale, comme dans discutable Conséquemment, pour pouvoir dire que le pseudo-mot parlable (base verbale) est plus probable que le pseudo-mot pontable (base nominale), il faut que le lecteur ait acquis, généralement 
de façon implicite, les règles de formation des mots multimorphémiques. Vraisemblablement ces règles se développent notamment lors des contacts du lecteur avec l'écrit (Gombert, 2003).

\section{Méthodologie}

\section{Les participants}

Parmi les 40 élèves sourds sévères ou profonds inscrits, au moment de l'étude, dans un milieu gestuel montréalais (Québec) qui n'accusaient pas plus de deux ans de retard scolaire et n'avaient aucun handicap associé, nous avons retenu les élèves les plus forts et les élèves les plus faibles en lecture suite à une épreuve informatisée de compréhension en lecture (Ciesielski et Reinwein, 1989). Au total, 24 élèves atteints d'une surdité sévère ou profonde âgés de 10 à 18 ans provenant de classes spéciales ont participé à cette étude. Ils ont été regroupés en fonction de leur âge et de leur niveau de lecture. Trois groupes d'âge ont été formés correspondant aux élèves de 10 à 12 ans, de 13 à 15 ans et de 16 à 18 ans. Dans la pratique, ces groupes d'âge correspondent au dernier cycle du primaire et aux premier et deuxième cycles du secondaire. Une analyse de variance robuste (Conover et Iman, 1981) a permis de justifier a posteriori la formation des sous-groupes. Cette analyse révèle un effet de l'âge et du niveau de lecture. Aucune interaction entre l'âge et le niveau de lecture n'est observée. En d'autres mots, les sujets de 10-12 ans ont une performance inférieure aux sujets de 13-15 ans qui, euxmêmes, réussissent moins bien que les sujets de 16-18 ans et les sujets faibles en lecture ont des résultats moins élevés que les sujets forts.

\section{Le matériel expérimental}

De façon générale, les études présentées jusqu'ici ont eu recours à un matériel expérimental formé de mots dans le cadre d'épreuves de lecture à voix haute ou encore de décision lexicale. Compte tenu de la population visée par la présente étude, une épreuve de lecture à voix haute est inadé- 
quate. Par ailleurs, l'utilisation d'une tâche de décision lexicale pose aussi problème. En effet, le recours à des mots implique la prise en compte de nombreux facteurs : fréquence de base et de surface des mots, structure des mots, connaissances lexicales préalables des sujets. Or, comme nous l'avons souligné, les jeunes sourds ont souvent des connaissances lexicales faibles. Par ailleurs, puisqu'un des objectifs de cette étude, comme nous le verrons plus loin, est de comparer des sujets d'âges différents, il devenait difficile d'utiliser le même matériel expérimental auprès de tous les sujets. Nous avons donc opté pour une épreuve de jugement de probabilité lexicale. Cette épreuve, semblable à celle utilisée par Burani et al. (2002), ne demande pas aux sujets de déterminer si les items écrits sont ou non des mots, mais plutôt s'ils sont ou non probables.

Le matériel expérimental est formé de 56 paires de pseudo-mots dont l'un respecte les règles de formation des mots en français (parlable) et l'autre non (pontable). Les pseudo-mots sont tous formés à partir d'une base lexicale et d'un affixe réels. Les bases lexicales ont été choisies à partir des listes de vocabulaire prescrit pour le premier cycle du primaire (élèves de 7 à 9 ans) par la commission scolaire, c'està-dire le regroupement des écoles où les sujets sont inscrits (CÉCM, 1992). Les mots de ces listes font l'objet d'un enseignement explicite et sont donc censés être connus des lecteurs. Nous avons opté pour les listes du premier cycle du primaire afin de nous assurer que les bases lexicales utilisées pour la formation des pseudo-mots seraient reconnues par tous les sujets et afin d'éviter la variabilité au niveau de la reconnaissance de ces bases qui aurait pu être causée par leur fréquence respective. Les affixes ont été sélectionnés en fonction de leur fréquence dans la langue et des règles de formation des mots (Bosquart, 1998 ; Grevisse, 1993). Les affixes choisis font partie des affixes les plus productifs du français, sans toutefois être nécessairement les plus fréquents. Les suffixes choisis sont -age et -able (donnage - dentage; tournable - tristable). Les préfixes retenus sont pré- et re(prédonner-prédouce; reboire - rebonne). Les items expérimentaux sont présentés en annexe. Dans ces exemples, le premier item de chaque paire est probable, c'est-à-dire qu'il res- 
pecte les règles de formation des mots du français, et le second improbable. Pour chaque affixe, 14 paires de pseudomots ont été formées. Les bases des pseudo-mots d'une même paire ont toujours la première lettre en commun. Pour un affixe donné, les bases n'apparaissent jamais deux fois dans une même liste. De plus, le nombre de lettres formant les pseudo-mots a été pris en compte.

Un taux de réussite supérieur au hasard, illustré par une préférence pour les items probables en français, indiquerait que les sujets ont accès aux règles de formation des mots et donc que le morphème est susceptible d'être une unité de lecture pour ces sujets. Par ailleurs, une différence significative $(p<0,05)$ entre les résultats portant sur les items suffixés et ceux portant sur les items préfixés pourrait indiquer une plus grande difficulté à extraire les règles de formation de mots dans l'un ou l'autre cas.

\section{Procédures}

Nous avons eu recours au logiciel LÉA (Bastien, 2002) pour concevoir le matériel d'évaluation de cette expérimentation. Chaque paire d'items est présentée de la même façon. Les deux items écrits en lettres minuscules apparaissent côte à côte au milieu de l'écran. La position de la réponse attendue est đéérinie aléatoirement. La consigne est la suivante : Détermine lequel des deux items est le plus probable en français écrit. Pour répondre, le sujet doit appuyer sur une des deux touches définies préalablement et bien identifiées sur le clavier de l'ordinateur qui correspondent aux choix de réponses. Après l'enregistrement de la réponse (maximum $10000 \mathrm{~ms}$ ), les deux items disparaissent de l'écran. Une suite de XXXX apparait après $1000 \mathrm{~ms}$ et demeure à l'écran pendant 1000 ms. Une autre paire devient visible à l'écran après un intervalle de $1000 \mathrm{~ms}$. L'épreuve débute par 5 paires d'entrainement suivies des 56 paires expérimentales regroupées en blocs de 5 paires. Une pause d'une durée non déterminée est prévue entre chaque bloc. Le sujet contrôle la durée de la pause en appuyant sur la barre d'espacement pour activer le bloc suivant. 
Dans le cadre de la validation des procédures expérimentales, le matériel a d'abord été utilisé auprès d'élèves entendants du même âge que les sujets sourds de cette étude et auprès d'adultes sourds. A l'hiver 2003, les sujets sourds ont été évalués individuellement dans une pièce calme et une expérimentatrice sourde a donné les explications en langue des signes québécoise.

\section{Résultats}

Des analyses inférentielles ont été menées pour déterminer les éventuels effets de l'âge et du niveau de lecture sur la réussite. Les moyennes des pourcentages de réussite, déterminées par la sélection des items probables en français, sont présentées, pour chaque groupe de sujets, à la Figure 1.

Une analyse de variance révèle un effet de l'âge : les sujets du groupe d'âge 1 (10 à 12 ans) obtiennent des scores significativement plus bas que les sujets des groupes d'âge 2 et 3 (13 à 15 ans et 16 à 18 ans), lesquels ne se différencient pas entre eux. Un effet du niveau de lecture est aussi noté : les sujets faibles en lecture ont des résultats plus faibles à cette épreuve que les sujets forts en lecture. Aucune interaction significative entre ces deux variables ne ressort de l'analyse. Compte tenu du nombre de sujets, nous considérons cependant l'interaction tendancielle, ce qui expliquerait pourquoi la différence entre les sujets faibles en lecture des groupes d'âge 2 et 3 est beaucoup moins marquée que celle se rapportant aux sujets forts des mêmes groupes d'âge $(0,45 \%$ et $13,39 \%$, respectivement).




Nous avons aussi tenté de déterminer si le type d'affixe influençait les résultats des sujets. Les résultats de l'analyse de variance indiquent que le pourcentage de réussite pour les items préfixés (moyenne: $71,57 \%$ ) est significativement supérieur à celui pour les items suffixés (moyenne: $65,77 \%$ ). Cet effet du type d'affixe s'applique à l'ensemble des sujets puisque l'âge, le niveau de lecture ou l'interaction entre les deux variables n'expliquent pas la différence entre les items préfixés et suffixés.

Enfin, nous avons tenu à prendre en compte le hasard étant donné que les choix de réponse étaient tous binaires avec une probabilité de succès de $50 \%$. Le modèle statistique utilisé pour évaluer le taux de hasard de la somme des réponses est une distribution multinomiale. Nous avons déterminé la limite de niveau $95 \%$ de la borne supérieure, c'est-à-dire le pourcentage de réussite au-delà duquel les résultats ne sont plus attribuables au hasard. Le score correspondant à la borne supérieure est de $60,7 \%$. Avec des scores se situant au-delà de $70 \%$, les sujets des groupes d'âge 2 et 3 ne semblent vraisemblablement pas répondre au hasard. Par contre, les réponses des sujets faibles en lecture du groupe d'âge 1 ne se distinguent pas du hasard et celles des sujets forts en lecture du groupe d'âge 1 sont attribuables au hasard dans la moitié des cas. L'analyse des effets du hasard tend à montrer que ces résultats sont fidèles, indiquant ainsi que la majorité des sujets peuvent traiter la structure morphologique de pseudomots.

\section{Discussion et conclusion}

La majorité des sujets de cette étude ont induit certaines règles de formation des mots, vraisemblablement à partir de leur expérience de l'écrit puisque ces règles ne sont généralement pas enseignées à l'école québécoise. La prise en compte du degré de fidélité renforce ce constat. En effet, chez les élèves forts en lecture, les réponses ne semblent pas être attribuables au hasard dès le premier groupe d'âge (10-12 ans). Chez les élèves faibles en lecture les résultats peuvent être considérés fidèles dès le deuxième groupe d'âge (13-15 ans). 
Beauvillain (1994) et Taft (1994), chez des sujets adultes ainsi que Laxon et al. (1992) et Burani et al. (2002), chez de jeunes lecteurs entendants, avaient aussi noté cette sensibilité à la légalité morphologique observée dans la présente étude.

Par ailleurs, nos résultats indiquent un effet de l'âge. Plus les sujets sont âgés, meilleurs sont les taux de réussite. Singson et al. (2000), Casalis et Louis-Alexandre (2000) et Burani et al. (2002) avaient aussi relevé, chez les entendants, un effet de l'âge dans leur étude. De plus, nous obtenons, comme Laxon et al. (1992) et Yu-Min et Anderson (2003) un effet du niveau de lecture. Dans le cas de la présente étude, les effets de l'âge et du niveau de lecture indiquent que plus les lecteurs sont âgés et plus élevé est leur niveau de lecture, plus ils sont aptes à identifier les items écrits qui respectent les règles de formation des mots du français.

Toujours dans la présente recherche, la différence significative entre les taux de réussite pour les items préfixés et suffixés qui se manifeste pour tous les sujets est intéressante. Premièrement, puisque ces taux moyens de réussite sont audessus du seuil du hasard, il faut conclure qu'un traitement morphologique a eu lieu, peu importe le type d'affixe $(71,57 \%$ pour les items préfixés et $65,77 \%$ pour les items suffixés). Cependant, il semble que les sujets aient mieux réussi à extraire les règles de formation pour les items préfixés que pour les items suffixés. Pour expliquer cette différence, nous faisons l'hypothèse que dans le cas des items suffixés, la présence de l'image complète d'un mot dans la réponse non attendue a pu nuire au jugement des sujets. En effet, les items suffixés correspondant aux règles de formation des mots ne comportaient pas de mot (frapp dans frappage n'est pas un mot) alors que ceux qui ne respectaient pas les règles de formation des mots incluaient toujours un mot (forêt dans forêtage). La présence d'un mot connu comme forêt a donc pu induire les sujets en erreur. Si tel est le cas, il faudra conclure que le traitement sublexical de type morphologique (l'analyse des composantes morphologiques du mot) observé est entravé par la présence d'un traitement lexical (analyse du mot de façon globale). Il serait intéressant de vérifier cette hypothèse dans le cadre d'autres études ayant recours à d'autres paradigmes expérimentaux. 
En conclusion, les résultats de cette étude indiquent que les élèves sourds plus âgés et ceux dont le niveau de lecture est plus élevé sont en mesure de traiter l'information morphologique contenue dans des pseudo-mots. Bien évidemment, ces conclusions ne s'appliquent qu'au cadre défini dans ce travail et ne peuvent être généralisées à tous les contextes de lecture. Il n'en demeure pas moins qu'il s'agit des premiers résultats de recherche à montrer que les sourds peuvent traiter l'information morphologique et développer une sensibilité aux règles de formation de mots. Ces résultats semblent cependant suggérer que la capacité à prendre en compte les indices morphologiques se développe plus tard chez les sourds (à partir de 12 ans dans cette étude) que chez les entendants (voir résultats des études présentés plus haut). Il est possible que le protocole méthodologique retenu pour cette étude soit responsable de cette différence. Pour cette raison, d'autres études, où élèves sourds et élèves normo-lecteurs sont comparés, seront nécessaires pour bien comprendre le rôle de la structure morphologique des mots sur leur reconnaissance.

Il ressort enfin de cette étude que le traitement sublexical de nature morphologique semble être en compétition, chez les sourds, avec un traitement lexical. Compte tenu du fait que la didactique de la lecture chez les sourds s'inspire grandement de l'approche whole-language (approche globale) développée pour l'enseignement de la lecture chez les entendants, il se peut que les comportements observés soient le reflet du type d'enseignement que reçoivent les élèves sourds. Si cette hypothèse était vérifiée dans le cadre d'autres études, cela aurait une incidence sur la façon d'aborder le traitement des mots lors de l'enseignement de la lecture chez les sourds. En effet, il faudrait alors amener les lecteurs à prendre davantage conscience de la nature segmentale des mots en développant des activités facilitant notamment la sensibilisation au traitement sublexical de type morphologique. Dans le domaine de la didactique de la lecture chez les sourds, la mise en place et l'évaluation de l'impact de telles activités sur le développement de la réussite en lecture constituent une des avenues de recherche importante à explorer dans les années qui viennent afin de mieux répondre aux besoins de ces élèves. 


\section{Références}

Abu-Rabia, S., Share, D. et Mansour, M. S. (2003) : Word Recognition and Basic Cognitive Processes among Reading-Disabled and Normal Readers in Arabic, Reading and Writing: An Interdisciplinary Journal, 16, 423-442.

BACCINo, T. et COLÉ, P. (1995) : La lecture experte, Paris, Presses Universitaires de France.

BASTIEN, M. (2002) : LÉA : le logiciel d'évaluation des apprentissages, Montréal, Université du Québec à Montréal, Département de linguistique et de didactique des langues.

Beauvillain, C. (1994) : Morphological Structure in Visual Word Recognition : Evidence from Prefixed and Suffixed Words, Language and Cognitive Processes, 9, 317-339.

BOSQUART, M. (1998) : Nouvelle grammaire française, Montréal, Guérin.

Burani, C. et Caramazza, A. (1987) : Representation and Processing of Derived Words, Language and Cognitive Processes, 2, 217-227.

Burani, C., Marcolini, S. et Stella, G. (2002) : How Early Does Morphological Reading Develop in Readers of a Shallow Orthography? Brain and Language, 81, 568-586.

Burani, C., Salmaso, D. et Caramazza, A. (1984) : Morphological Structure and Lexical Access, Visible Language, $18,342-352$.

Casalis, S. et Louis-Alexandre, M.-F. (2000) : Morphological Analysis, Phonological Analysis and Learning to Read French : A Longitudinal Study, Reading and Writing : An Interdisciplinary Journal, 12, 303-335.

CÉCM (1992) : Des mots pour écrire (1 ${ }^{r e}, 2^{e}$ et $3^{e}$ années du primaire), Montréal, Commission des écoles catholiques de Montréal.

Ciesielski, R. et Reinwein, J. (1989) : Le test Zigzag (version 2.0 Lightspeed Pascal), Montréal, Université du Québec à Montréal, Département de linguistique.

Colé, P., Beauvillain, C. et Segui, J. (1989) : On the Representation and Processing of Prefixed and Suffixed Derived Words : A Differential Frequency Effect, Journal of Memory and Language, 28, 1-13. 
Conover, W.J. et Iman, R.L. (1981) : Rank Transformation as a Bridge between Parametric and Nonparametric Statistics, The American Statistician, 35, 124-129.

GOMBERT, J.-É. (1997) : Les activités cognitives en œuvre dans la lecture et son acquisition, in F. Andrieux, J.M. Besse et $\mathrm{B}$. Falaize (dir.), Illettrisme : quels chemins vers l'écrit? Paris, Magnard, 129-144.

GOMBERT, J.-É. (2003) : L'apprentissage des codes graphophonologique et grapho-sémantique en lecture, in M.N. Romdhane, J.É. Gombert et M. Belajouza (dir.), L'apprentissage de la lecture - Perspectives comparables, Rennes, Presses Universitaires de Rennes, 19-34.

GREVISSE, M. (1993) : Le bon usage, Paris, Duculot.

HANSON, V.L. et WILKENFELD, D. (1985) : Morphophonology and Lexical Organization in Deaf Readers, Language and Speech, 28, 269-280.

LAXON, V., Rickard, M. et COlTHEART, V. (1992) : Children Read Affixed Words and Non-Words, British Journal of Psychology, 83, 407-423.

Paul, P. V. (1998) : Literacy and Deafness: The Development of Reading, Writing, and Literate Thought, Toronto, Allyn and Bacon.

Pollatsek, A., Hyönä, J. et Bertram, R. (2000) : The Role of Morphological Constituents in Reading Finnish Compound Words, Journal of Experimental Psychology : Human Perception and Performance, 26, 820-833.

Singson, M., Mahony, D. et ManN, V. (2000) : The Relation between Reading Ability and Morphological Skills : Evidence from Derivational Suffixes, Reading and Writing : An Interdisciplinary Journal, 12, 219-252.

SPRenger-Charolles, L. et Casalis, S. (1996) : Lire - lecture et écriture : acquisition et troubles du développement, Paris, PUF.

Stanovich, K. E. (1990) : Concepts in Developmental Theories of Reading Skill : Cognitive Resources, Automaticity, and Modularity, Developmental Review, 10, 72-100.

TAFT, M. (1994) : Interactive-Activation as a Framework for Understanding Morphological Processing, Language and Cognitive Processes, 9, 271-294. 
TRANSLER, C. (1999) : La lecture chez les enfants sourds sévères et profonds, analyse des traitements phonologiques et orthographiques, Thèse de doctorat, Université Libre de Bruxelles et Université de Haute Bretagne.

Yu-Min, K. et ANDERson, R. C. (2003) : Development of Morphological Awareness in Chinese and English, Reading and Writing: An Interdisciplinary Journal, 16, 399422.

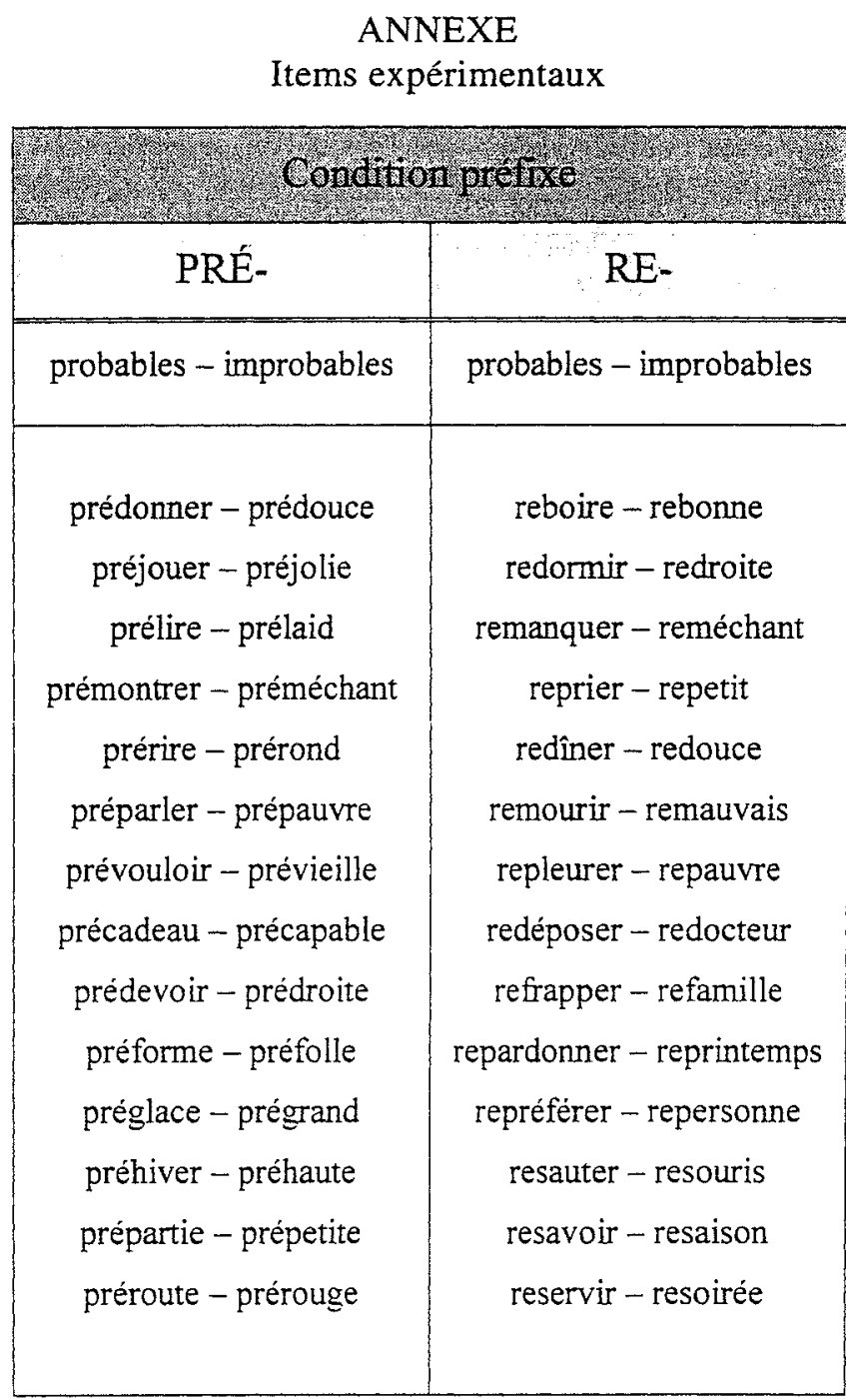




\begin{tabular}{|c|c|}
\hline -ABLE & -AGE \\
\hline probables - improbables & probables - improbables \\
\hline tournable - tristable & frappage - forêtage \\
sautable - seulable & jouage - jeuage \\
marchable - mauvaisable & montrage - métroage \\
glissable - grandable & préparage - papierage \\
frappable - froidable & présentage - poussinage \\
cherchable - chaudable & sonnage - soirage \\
battable - brunable & trouvage - tempsage \\
préparable - papierable & cherchage - chaudage \\
parlable - pontable & demandage - droitage \\
oubliable - ouestable & marchage - mauvaisage \\
montable - midiable & parlage - petitage \\
levable - litable & pensage - proprage \\
apportable - argentable & racontage - rondage \\
sautage - seulage
\end{tabular}

